\title{
Effects of Multi-symbols on Enhancing Virtual Reality Based Collaborative
}

\author{
Shih-Ching Yeh ${ }^{1}$, Wu-Yuin Hwang ${ }^{2, *}$, Jing-Liang Wang ${ }^{1}$, and Yuin-Ren Chen ${ }^{1}$ \\ ${ }^{1}$ Department of Computer Science and Information, National Central University, Taiwan \\ ${ }^{2}$ Institute of Network Learning Technology, National Central University, Taiwan \\ wyhwang1206@gmail.com
}

\begin{abstract}
Extended Summary. Applying virtual reality (VR) technologies to enhance learning becomes more and more popular. This research intends to investigate how multi-symbolic representations could help users being aware of collaborative context and partner's needs to enhance completing haptics-based collaborative tasks in a co-located/distant virtual environment. This study evaluates the performance of collaboration including the completing time and the number of failure in completing a task. To make users being aware of context, multisymbolic representations in forms of color and text are provided as well as haptics and audio feedback in the virtual environment. Participants in the experiment were separated into four groups with the combinations of two variables: w/o multi-symbols and co-located/distant. The results show that multi-symbols significantly helped users reduce the time in completing a task in the case of colocated collaborative virtual environment. However, there was no significant improvement in performance in the case of distant collaborative virtual environment. From our on-site observations, while operating the task collaboratively, several interesting behaviors existing between participants, such as strategy toward task success or failure, were found. First, after a few trials of completing the task in the beginning, instead of pinching and lifting the virtual cube directly toward the cone-like target, participants first push the cube and slide it to the underneath of the cone-like target, and then lifted it upward till reaching the target. Namely, participants were able to develop a good strategy with less completing time or errors therefore to complete the task more efficiently and successfully even though it was in a virtual environment. Second, the failure in completing the task was generally caused by inconsistent and incoordinate movements or force between partners. While intending to pinch the virtual cube, a balanced force output from left side and right side is required to prevent the sliding. Furthermore, to investigate how multi-symbols could affect user's perceptions, we investigate the perceived awareness, presence and social presence of our proposed system and its influence on perceived usefulness, ease of use and playfulness based on Technology Acceptance Model. The results showed that awareness, presence and social presence significantly influenced perceived usefulness, ease of use and playfulness. Therefore, our proposed multi-symbols virtual reality system has potentials to help collaborative learning.
\end{abstract}

Keywords: Representations, Virtual Reality Collaboration, Social presence.

\footnotetext{
* Corresponding author.
} 